\title{
Ossa Sesamoidea - prevalence of sesamoid bones in human hands
}

\author{
K. Dąbrowski , H. Stankiewicz-Jóźwicka², A. Kowalczyk¹, M. Markuszewski , B. Ciszek11,4 \\ ${ }^{1}$ Department of Descriptive and Clinical Anatomy, Medical University of Warsaw, Poland \\ 2Department of Piano, Harpsichord and Organ, The Fryderyk Chopin University of Music, Warsaw, Poland \\ ${ }^{3}$ Department of Choir Conducting, Music Education, Church Music, Rhythmics and Dance, \\ The Fryderyk Chopin University of Music, Warsaw, Poland \\ ${ }^{4}$ Department of Neurosurgery in Bogdanowicz Children's Hospital, Warsaw, Poland
}

[Received: 2 September 2019; Accepted: 21 October 2019]

\begin{abstract}
Background: This study describes the morphology of sesamoid bones in the human hand. Ultrasound imaging was used to record the presence and measurements of sesamoids in 120 hands of 60 healthy, young adults of Caucasian ethnicity.

Materials and methods: The mean number of sesamoid bones was 4.16 in the left hand and 4.03 in the right hand. $21.6 \%$ of cases showed asymmetry between the right hand and the left. There was a significant difference in the prevalence of sesamoid bones between right and left hand in males in this study. Females show a higher incidence of sesamoid bones overall, but do not demonstrate a significant difference between the sides.

Results: Prevalence findings: Two in the $1^{\text {st }}$ metacarpophalangeal joint (MCPJ) in $99.2 \%$ of cases, with 1 case of a single bone. One in the $2^{\text {nd }}$ MCPJ in $43.3 \%$ of cases. One in the $5^{\text {th }}$ MCPJ in $84.2 \%$ of cases. One in the $1^{\text {st }}$ interphalangeal joint (IPJ) in $83.4 \%$ of cases. Aside from that there was 1 case of sesamoid in the $3^{\text {rd }}$ MCPJ and separate case of sesamoid in the $3^{\text {rd }}$ proximal IPJ.

Conclusions: Moreover, this study discusses discrepancies of opinion regarding sesamoid bones in morphological research. (Folia Morphol 2020; 79, 3: 570-575)
\end{abstract}

Key words: sesamoids, palm, morphology, ultrasound, pattern

\section{INTRODUCTION}

Ossa Sesamoidea, or the sesamoid bones, are being described as small, oval shaped bony structures that normally form within the joint capsules or within or under tendons, usually above a joint. First description comes from second-century works of Galen, translated into Latin by Sylvius in 1561 under the title "De Ossibus Ad Tirones". In his work Galen describes small bones he discovered, most likely during the autopsy of a macaque monkey, that he compares in appearance to the seeds of Sesamum Indicum, which was used by ancient Greeks as a purgative. Versalis, on the other hand, in his "De Humani Corporis Fabrica Libri Septem" of 1542 compares the sesamoid bones to grains of rice or halves of peas and mentions a trend among his contemporary pseudo-scholars to assign to some of them an occult meaning $[2,8,10$, 19, 21, 22].

Modern descriptions of the sesamoid bones come from 1982 and at the very beginning of $20^{\text {th }}$ century the first pathologies of sesamoid bones were described [2, 22]. 
According to most authors describing prevalence of sesamoid bones in the human hand, there are at least two of them located in the $1^{\text {st }}$ metacarpophalangeal joint (MCPJ) that appear in $99-100 \%$ of population. A sesamoid appears in the $2^{\text {nd }}$ and/or $5^{\text {th }}$ MCPJ in around $50 \%$ of population just as the sesamoid in the $1^{\text {st }}$ interphalangeal joint (IPJ). That last one, however, seems to be strongly linked to the ethnicity of the examined population. Documentation of occurrences of sesamoids in the $3^{\text {rd }}$ and $4^{\text {th }}$ MCPJ is very rare $[2,8-11,14,17,19,22]$.

The exact function of sesamoid bones remains to be discovered. Some authors believe them to be evolutionary relics in regression, others - hereditary adjustments. The entire matter is complicated by findings that there are many more proto-sesamoids in the foetus than appear to calcify in the adult $[15,16$, 22]. Samuels et al. [12] observed that across the entire process of evolution of mammals, even as significant sesamoid as the Patella seems to have appeared and disappeared multiple times. Jones points out that in some contemporary mammals, for example rabbits, their equivalent of two contiguous sesamoid bones is actually one, dumb-bell shaped bone, with the flexor tendons coming over "the handle" part as if it was a pulley [6]. It is commonly acknowledged that they have a role in friction reduction, protection of the tendons, reduction of compression on the joint and modifying the direction of pull across the underlying joint $[1,4,7,8,10,13,22]$.

\section{MATERIALS AND METHODS}

The study recruited 120 hands of 60 healthy volunteers of Caucasian ethnicity ( 29 male and 31 female) between the age of 18 and 38 (mean: 23.2 years), 51 right handed and 9 left handed ( 5 male and 4 female) with no previous history of serious injury or surgical procedures in the area of the hand.

All participants were questioned regarding their history of injuries and surgical procedures, had opportunity to ask any questions they deemed relevant and signed the form of informed consent.

The hands were examined by ultrasound (LOGIQ F8 GE with L6-12, 6-13 MHz probe) for the presence of sesamoid bones on the palmar aspect in the proximity of MCPJs and IPJs (both proximal and distal). Structures recognised as sesamoid bones were measured and mapped on a pre-prepared stencil of the hand, with regard of their location in the hand and their measurements: the anterior-posterior (AP) measurement, the ulnar-radial measurement (the probe located perpendicularly to the longitudinal axis of the examined finger) and the proximal-distal measurement (the probe located parallelly to the longitudinal axis of the examined finger).

\section{Statistical analysis}

All the findings were examined with use of appropriate statistical tests in Microsoft Excel pack and auxiliary internet tools - Fisher's Exact test for the unpaired variables, Student's t-test for paired variables and Grubbs' test for an outlier for singular cases - with constant assumption of $\alpha=0.05$.

Due to complete non-invasiveness of the examination, approval of Ethics Committee was not necessary.

\section{RESULTS}

The number of sesamoid bones present varied from 2 to 6 per hand, with a mean prevalence being of 4.1 per hand $(4.16$ for the left hand and 4.03 for the right hand). No significant difference between right-handed and left-handed individuals was observed. Asymmetry in the pattern of sesamoid distribution was observed in 13 out of 60 (21.6\%) participants examined ( 7 female, 6 male). 20 out of $120(16.6 \%)$ examined hands lacked a sesamoid in the IPJ of the thumb (10 left hands and 10 right hands. 14 female - two of which unilaterally - and 8 male). A sesamoid bone was observed in MCPJ of the $2^{\text {nd }}$ finger in 52 out of $120(43.3 \%)$ examined hands (25 right -18 female and 7 male, and 27 left 17 female and 10 male, with 4 cases of asymmetry 1 female and 3 male). 101 cases out of 120 (84.2\%) presented with sesamoid bone in the MCPJ of the $5^{\text {th }}$ finger, with only 11 absences ( 5 male, 6 female) on the right side and 8 absences ( 3 male, 5 female) on the left side, out of which 5 ( 2 male and 3 female) were unilateral. On average females have more sesamoids than males and left hands have more sesamoids than right hands; however, only the left-right comparison in men (Student's t-test $p$-value $=0.005$ ) reaches the threshold of statistical significance in this analysis. In 1 case a sesamoid was observed in the MCPJ of the $3^{\text {rd }}$ finger (female, 24 years old, Grubbs' test Z-value $=1.36$, Critical Z-value $=3.88$ ). In 1 case a sesamoid was observed in the proximal IPJ of the $3^{\text {rd }}$ finger (male, 22 years old, Grubbs' test Z-value $=1.29$, Critical Z-value $=3.88$ ). There was also 1 case of unilateral singular sesamoid in the $1^{\text {st }}$ MCPJ on the right side (female, 26 years old, Grubbs' test Z-value $=0.82$ for Critical Z-value = 3.57) (Fig. 1). 


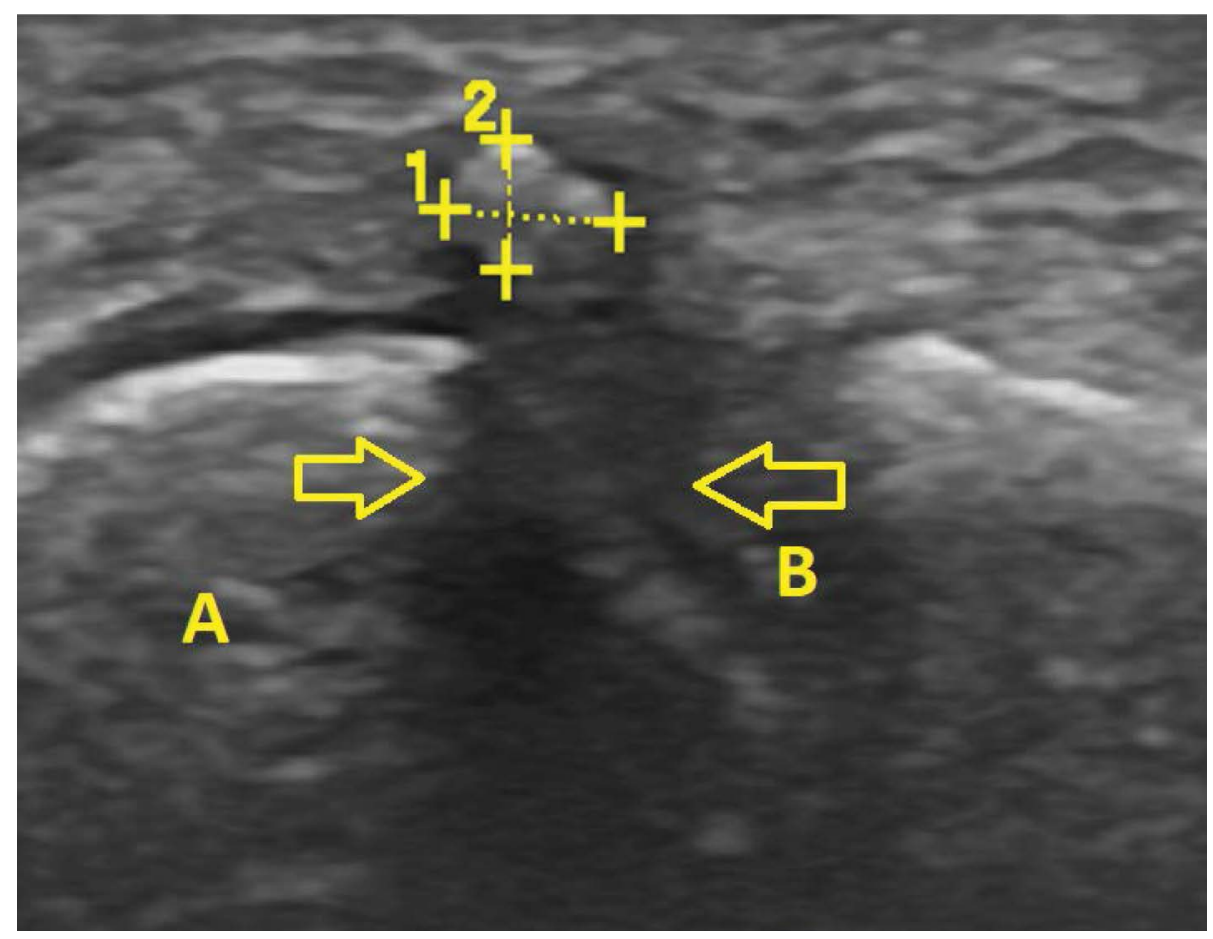

Figure 1. A sesamoid bone in the $5^{\text {nd }}$ metacarpophalangeal joint of the right hand of the 28 -year-old male - a sagittal plane view. The sesamoid is located above the articular fissure (arrows) between the basis of the $5^{\text {nd }}$ proximal digit $(A)$ and the head of the $5^{\text {nd }}$ metacarpal bone $(B)$. The measures noted are anterior-posterior $(2)=1.8 \mathrm{~mm}$ and proximal-distal $(1)=2.6 \mathrm{~mm}$.

\section{DISCUSSION}

Although there is a significant amount of existing research examining the prevalence of sesamoid bones, authors do not seem to agree regarding the results. There is some evidence that the location and number of sesamoids is hereditary [16], related to ethnicity $[8,17,19]$ and sex $[11,15,16,19]$, yet there are many discrepancies documented even within the same ethnic group. This may be explained by the frequent use of $X$-ray imaging as a sole research tool. Some authors question reliability of the X-ray imaging, especially classical AP projection. In some cases sesamoid bones, especially those in more unorthodox locations, are misdiagnosed as lose bone fragments $[13,22]$. Some other papers use $X$-ray to locate sesamoids in younger patients [8], while due to ossification beginning in the $1^{\text {st }} \mathrm{MCPJs}$ around the age of 10-12 for females and 12-15 for males, the results may be unreliable $[4,8,18,22]$.

The authors of this paper decided to use ultrasound imaging as it is potentially more reliable in the specific case of identifying and diagnosing sesamoid bones [1], as well as representing a more flexible and less invasive examination tool.

Many researchers seem to suggest that the presence of two sesamoids in the $1^{\text {st }} \mathrm{MCPJs}$ is a universal finding $[2,8,9,14,18,19]$, or even straight up assumes so from the very beginning $[15,16,17]$. Others refer to the aforementioned bones as "almost always present" or "present in over $99 \%$ cases" leaving a possibility of physiological agenesis open $[1,3,10,11,22]$. There are also reported cases of complete absence of sesamoid bones, presence of just one or presence of three sesamoid bones in the $1^{\text {st }} \mathrm{MCPJ}[8,11]$. In this research there was only 1 case of single sesamoid bone in $1^{\text {st }}$ MCPJ, which dimensions, according to Grubbs' test for an outlier, fall within the norm for sesamoid bones (Grubbs' Z-value $=0.82$ with Critical $Z$-value $=3.57$ ), which undermines the theory that two sesamoid bones may have coalesced into one. The lack of trauma or surgical procedures history excludes the possibility of a previous extraction of one sesamoid bone.

According to existing literature, Os Sesamoideum should be present in $2^{\text {nd }}, 3^{\text {rd }}, 4^{\text {th }}$ and $5^{\text {th }}$ MCPJ in $36.6-60.8 \%, 1.5-3.9 \%$, below $0.6-2 \%$ and $45-67.7 \%$ of cases, respectively, with supposed ethnical tendency for $2^{\text {nd }}$ and $5^{\text {th }}$ MCPJ sesamoid bones being more frequent in Arabic and Mediterranean population and less frequent in African population [2, 8-11, 14-17, $19,22]$. There are also mentions of the records describing "double" sesamoid bones in $1^{\text {st }}$ and $5^{\text {th }}$ MCPJ [2]. 


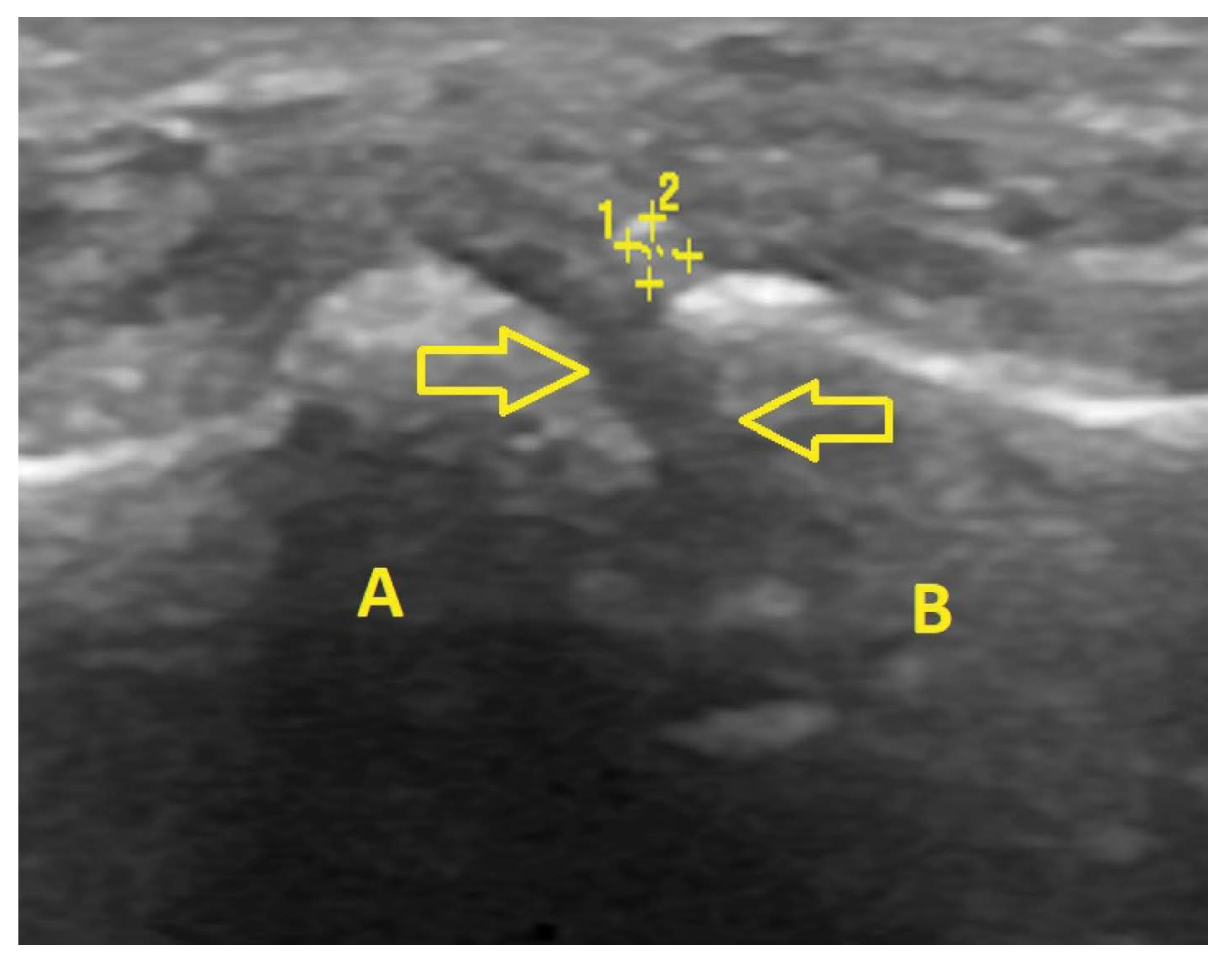

Figure 2. A sesamoid bone in the $3^{\text {rd }}$ proximal interphalangeal joint of a left hand of a 22-year-old male - a sagittal plane view. The sesamoid is located right above the articular fissure of the $3^{\text {rd }}$ proximal interphalangeal join (arrows) between the basis of the $3^{\text {rd }}$ medial digit $(A)$ and the head of $3^{\text {rd }}$ proximal digit $(B)$, probably within the walls of the articular capsule. The measures noted are anterior-posterior $(2)=0.9 \mathrm{~mm}$ and proximal-distal $(1)=1.0 \mathrm{~mm}$.

In this research out of 120 examined hands there were 52 sesamoid bones observed in the $2^{\text {nd }} \mathrm{MCPJ}$ ( $43.3 \%$ prevalence) -25 in the right hand ( 18 female, 7 male) and 27 in the left hand (17 female, 10 male) with 4 cases of asymmetry ( 1 female, 3 male). There was only 1 case of sesamoid bone present in the $3^{\text {rd }}$ MCPJ $(0.8 \%)$ and no cases in the $4^{\text {th }}$ MCPJ. In the $5^{\text {th }}$ MCPJ there were 101 sesamoids (84.1\%) observed, 49 in the right hand ( 24 female, 24 male) and 52 in the left hand (25 female, 26 male) with 5 cases of asymmetry (3 female, 2 male).

The sesamoid bone in $1^{\text {st }}$ IPJ is the only frequently observed sesamoid in an interphalangeal joint. According to the literature, it is present in $21.3-75 \%$ of cases [8-11, 14-17, 19, 21] while being heavily dependent on ethnicity (more prevalent in the African population and less prevalent in Arabic and Middle Eastern populations) $[8,17,19]$ and sex (more frequent in females) $[16,22,23]$. These results do not allow us to form any clear expectations regarding situation in vivo. Chen et al. [2] reported prevalence of Os Sesamoideum in $1^{\text {st }}$ IPJ at $15.9 \%$ which the authors themselves describe as surprisingly low, particularly in comparison to existing research setting the prevalence of sesamoids in that joint at $100 \%$. In this research the sesamoid bone in the $1^{\text {st }}$ IPJ was observed in 100 out of 120 (83.3\%) cases, 50 in the right hand and 50 in the left hand with only 2 cases of asymmetry (both female).

Sesamoid bones in the rest of the interphalangeal joints remain very rare. There are singular cases recorded of presence in the $2^{\text {nd }}$ proximal IPJ $(0.4 \%$ at most), $3^{\text {rd }}$ proximal IPJ ( $0.4 \%$ at most), $4^{\text {th }}$ proximal IPJ $(0.5 \%$ at most $)$ and $5^{\text {th }}$ proximal IPJ ( $1 \%$ at most) [11, 13,22 ]. Kose et al. [8] observed a sesamoid bone in the $2^{\text {nd }}$ distal IPJ. In this research there was observed only 1 case - of sesamoid in the $3^{\text {rd }}$ proximal IPJ.

The measurements of sesamoid bones that were identified in the $3^{\text {rd }}$ MCPJ and in the $3^{\text {rd }}$ proximal IPJ (two separate cases) were compared to the measurements of the other observed sesamoids. Their results were Grubbs' Z-value $=1.36$ and Grubbs' Z-value $=1.29$, respectively with assumed standard $\alpha=0.05$ and Critical Z-value of 3.88. This suggests that, considering their location above the finger joints, measurements and echogenicity falling within the norm for sesamoids for the examined group, they must be considered legitimate sesamoid bones (Fig. 2).

The matters of symmetry and sexual dimorphism remain divisive in the existing research. Most authors agree that sesamoid bones are more frequently found 
in the hands of females, although some, who draw connection between prevalence of sesamoids and manual labour, seem puzzled by that finding. Many studies have found this difference to be statistically significant $[8,16]$ while others report it insignificant $[11,19,22]$. Nevertheless, evidence suggests that even very experienced radiologists are unable to determine patients sex based on X-rays of their hands alone [23]. The matter of symmetry seems just as unclear. Some authors report that the distribution of Ossa Sesamoidea is always completely symmetrical [19]. Others recognise the possibility of asymmetry, but deem it an omittable rarity [8], or report that the asymmetry can appear only in "rare" cases when there are more than 3 sesamoids in the hand [11]. However, there are also papers claiming that asymmetry in the sesamoid pattern is real and significant - at the level of $17.1 \%$ [16].

This research found females to have on average more sesamoid bones than males, with $p$-value of Fisher's test close, but not within the values of statistical significance: $p$-value $=0.094$. It is possible that, given a larger sample, sexual dimorphism may prove to be statistically significant.

Regarding lateralisation, 13 out of 60 examined people $(21.7 \%)$ showed some form of asymmetry in the distribution of their sesamoid bones, which is not an omittable amount. On average sesamoid bones were more common in the left hand than in the right (mean of 4.16 to 4.03). T-Student's test for the entire examined group showed $\mathrm{p}$-value $=0.204$ which is not significant. However, when split by sex, the results were $p$-value $=0.211$ for the females and $p$-value $=0.005$ for the males - the latter being well within the range of statistical significance.

The exact function of Ossa Sesamoidea remains to be discovered and described. Most popular theories suggest that Ossa Sesamoidea function of reduce friction of the sliding tendons across the underlying structures, therefore protecting them from use-related damage. Observing how these bones are being shaped by compression of the tendons suggests that they reduce the pressure on the adjoined bones. Other possible functions include stabilisation and strengthening of the joint, fixing tendons in their place and modifying the direction of a muscle pull vector, therefore making the specific movement faster, stronger and less energy-consuming $[2-5,7,8,10$, $13,14,19,22]$.

The clinical importance of sesamoid bones comes both from their potential function as important land- marks, for example in corticosteroid injection procedures [20], and their pathologies. Ossa Sesamoidea can be the subject of trauma, degenerative diseases, neoplasia and necrosis [10]. According to some studies up to $85 \%$ of adults can suffer from some level of arthritis of the sub-sesamoid joints [22]. However, the most common pathology seems to be sesamoid fracture caused by forced hyperextension of the finger. First documented in 1905 in Germany [22], this injury can present a tenderness and swelling of the area of the joint without affecting either active or passive range of motion. In some cases a sesamoid fragment can completely lock an underlying joint. Due to its inscrutability, incompatibility with an X-ray imaging and the variability of symptoms, many pathologies of sesamoid bones remain misdiagnosed, and therefore mistreated [1, 10, 13, 22].

\section{CONCLUSIONS}

This study reports the prevalence of Ossa Sesamoidea in the hands of young adults of Caucasian ethnicity. It confirms possibility of singular sesamoid bone in the $1^{\text {st }}$ MCPJ. The presence of the sesamoid bones in $1^{\text {st }} \mathrm{IPJ}$ was $82.2 \%$ and in $2^{\text {nd }} \mathrm{MCPJ}$ was $43.3 \%$ which both matches the African pattern the closest, with former being higher than previously recorded prevalence. The sesamoid bone in $5^{\text {th }}$ MCPJ was present in $84.1 \%$ of cases which is closest to Arabic pattern and higher than previously recorded prevalence. The sesamoid bones were observed once in $3^{\text {rd }}$ MCPJ and once in $3^{\text {rd }}$ proximal IPJ and their measurements were statistically proven to match other sesamoid bones of the hand. $21.7 \%$ of examined cases showed asymmetry in the presence of sesamoid bones between the right and the left hand, which is higher than previously recorded occurrence.

\section{REFERENCES}

1. Becciolini M, Bonacchi G. Fracture of the sesamoid bones of the thumb associated with volar plate injury: ultrasound diagnosis. J Ultrasound. 2015; 18(4): 395-398, doi: 10.1007/s40477-015-0166-1, indexed in Pubmed: 26550076.

2. Chen W, Cheng J, Sun R, et al. Prevalence and variation of sesamoid bones in the hand: a multi-center radiographic study. Int J Clin Exp Med. 2015; 8(7): 11721-11726.

3. Dauwe J, Van der Cruyssen F, De Smet L, et al. Case report: Oblique fracture of the ulnar sesamoid bone of the metacarpophalangeal joint, a rare pathology. J Orthop Case Rep. 2017; 7(2): 29-32, doi: 10.13107/jocr.2250-0685.736.

4. Egerci OF, Kose O, Turan A, et al. Prevalence and distribution of the fabella: a radiographic study in Turkish sub- 
jects. Folia Morphol. 2017; 76(3): 478-483, doi: 10.5603/ FM.a2016.0080, indexed in Pubmed: 28026849.

5. Jin ZW, Shibata $S$, Abe $H$, et al. A new insight into the fabella at knee: the foetal development and evolution. Folia Morphol. 2017; 76(1): 87-93, doi: 10.5603/ FM.a2016.0048, indexed in Pubmed: 27665955.

6. Jones FW. The principles of Anatomy as seen in hand. 2nd ed. Bailliere Tindall and Cox 1994.

7. Koo BS, Song $Y$, Lee $S$, et al. Prevalence and distribution of sesamoid bones and accessory ossicles of the foot as determined by digital tomosynthesis. Clin Anat. 2017; 30(8): 1072-1076, doi: 10.1002/ca.22952, indexed in Pubmed: 28714106.

8. Kose O, Guler F, Turan A, et al. Prevalence and distribution of sesamoid bones of the hand: a radiographic study in turkish subjects. Int J Morphol. 2012; 30(3): 1094-1099, doi: $10.4067 / \mathrm{s} 0717-95022012000300055$.

9. Koo BS, Song Y, Sung YK, et al. Prevalence and distribution of sesamoid bones in the hand determined using digital tomosynthesis. Clin Anat. 2017; 30(5): 608-613, doi: 10.1002/ca.22881, indexed in Pubmed: 28340518.

10. Ozcanli $H$, Sekerci R, Keles N. Sesamoid disorders of the hand. J Hand Surg Am. 2015; 40(6): 1231-1232, doi: 10.1016/j.jhsa.2015.01.029, indexed in Pubmed: 25771478.

11. Rosengarten E. Occurence of Sesamoid bones in the hands of the Polish population. Folia Morphol. 1967: 2.

12. Samuels ME, Regnault $S$, Hutchinson JR. Evolution of the patellar sesamoid bone in mammals. PeerJ. 2017; 5: e3103, doi: 10.7717/peerj.3103, indexed in Pubmed: 28344905.

13. Seki Y, Hoshino Y, Kuroda H. Prevalence of sesamoid bones in the interphalangeal joint of the thumb and fingers: a radiographic study. Clin Anat. 2013; 26(7): 823-826, doi: 10.1002/ca.22201, indexed in Pubmed: 23255267.

14. Seki $Y$, Hoshino $Y$, Kuroda $H$. Locking of the ring finger at proximal interphalangeal joint due to a sesamoid bone: a case report. Hand (NY). 2012; 7(1): 119-120, doi: 10.1007/ s11552-011-9386-9, indexed in Pubmed: 23450978.

15. Sokołowska-Pituchowa J, Miaśkiewicz C. Studies on the Sesamoid bones in the hands of Men. Folia Morphol. 1965: 2.

16. Sokolowska-Pituchowa J, Miaśkiewicz C. [Studies on the sesamoid bones of the hands in women]. Folia Morphol. 1967; 26(1): 25-32, indexed in Pubmed: 5298792.

17. Sokołowska-Pituchowa J, Miaśkiewicz C. The Sesamoid bones in Negros. Folia Morphol. 1969: 2.

18. Szmurlo J, Szwaykowski W, Olczak A. Time of appearance of the ossification centre in the metacarpophalangeal sesamoid bone of the thumb in girls during puberty. Folia Morphol. 1975; 34(3): 259-265, indexed in Pubmed: 1080730.

19. Ting Lam GY, Chow E, Ng B, et al. Distribution of sesamoid bones in the hand - a study in the Chinese population. J Orthop Trauma Rehab. 2017; 23: 45-48, doi: 10.1016/j. jotr.2017.03.001.

20. Vargas A, Chiapas-Gasca K, Hernández-Díaz C, et al. Clinical anatomy of the hand. Reumatol Clin. 2012; 8 Suppl 2: 25-32, doi: 10.1016/j.reuma.2012.10.004, indexed in Pubmed: 23219083.

21. Versalis A. De Humani Corporis Fabrica Libri Septem: An annotated translation of 1542 and 1555 editions, Garrison DH, Hast MH, Karger 2014.

22. Yammine $K$. The prevalence of the sesamoid bones of the hand: a systematic review and meta-analysis. Clin Anat. 2014; 27(8): 1291-1303, doi: 10.1002/ca.22378, indexed in Pubmed: 24615762.

23. Yune $S$, Lee $H$, Kim $M$, et al. Beyond human perception: sexual dimorphism in hand and wrist radiographs is discernible by a deep learning model. J Digit Imaging. 2019; 32(4): 665-671, doi: 10.1007/s10278-018-0148-x, indexed in Pubmed: 30478479. 